Portland State University

PDXScholar

10-1-2009

\title{
Plasmonic enhancement of Forster energy transfer between two molecules in the vicinity of a metallic nanoparticle: nonlocal optical effects
}

\author{
P.T. Leung \\ Portland State University \\ H. Y. Xie \\ H. Y. Chung \\ D. P. Tsai
}

Follow this and additional works at: https://pdxscholar.library.pdx.edu/phy_fac

Part of the Physics Commons

Let us know how access to this document benefits you.

\section{Citation Details}

Xie, H. Y., Chung, H. Y., Leung, P. T., \& Tsai, D. P. (2009). Plasmonic enhancement of Forster energy transfer between two molecules in the vicinity of a metallic nanoparticle: nonlocal optical effects. Physical Review B (Condensed Matter And Materials Physics), 80(15), 155448.

This Article is brought to you for free and open access. It has been accepted for inclusion in Physics Faculty Publications and Presentations by an authorized administrator of PDXScholar. Please contact us if we can make this document more accessible: pdxscholar@pdx.edu. 


\title{
Plasmonic enhancement of Förster energy transfer between two molecules in the vicinity of a metallic nanoparticle: Nonlocal optical effects
}

\author{
H. Y. Xie (謝懷毅), ${ }^{1}$ H. Y. Chung (鍾弘毅), ${ }^{1}$ P. T. Leung (梁培德), ${ }^{1,2, *}$ and D. P. Tsai (蔡定平) ${ }^{1}$ \\ ${ }^{1}$ Department of Physics, National Taiwan University, Taipei, Taiwan 10617, Republic of China \\ ${ }^{2}$ Institute of Optoelectronic Sciences, National Taiwan Ocean University, Keelung, Taiwan 20224, Republic of China \\ (Received 13 March 2009; revised manuscript received 3 September 2009; published 26 October 2009)
}

\begin{abstract}
The problem of Förster resonance energy transfer (FRET) between two molecules in the vicinity of a metallic nanoparticle such as a nanoshell is studied within a phenomenological model which takes into account the nonlocal optical response of the metal. This model allows for arbitrary locations and orientations of the two molecular dipoles with respect to the metal particle which can be of ultrasmall sizes $(<10 \mathrm{~nm})$ and for which nonlocal effects are of high significance. In particular, for the nanoshell case, the molecules can be located both outside, both inside, or one inside and one outside the shell. Also, the case with a metallic spherical particle studied mostly in the literature can be obtained in the limit of zero inner radius for the nanoshell. Particular focus will be on the enhancement of this FRET process due mainly to the surface plasmon excitation of the free metallic electrons, and the nonlocal effects on this will be studied with reference to a number of factors including the molecular locations and orientations, the transition frequency of the donor and acceptor;...etc. Numerical results show that the resonances in the enhanced FRET rate will be dominated by the multipolar bonding and antibonding cross-coupled plasmonic modes of the nanoshell; and that the nonlocal effects will generally lead to blueshifted resonances, as well as diminution of the enhancement for the low-frequency portions of both modes. Such information will be useful for future application of plasmonic enhanced FRET using metallic particles of ultrasmall sizes.
\end{abstract}

DOI: 10.1103/PhysRevB.80.155448

PACS number(s): 78.67.Bf, 73.20.Mf, 41.20.Cv, 82.20.Ln

\section{INTRODUCTION}

Energy transfer between a molecule in an excited state (i.e., a donor) and one in ground state (i.e., acceptor) is an important fundamental process underlying many significant photophysical and photochemical processes from photosynthesis to fluorescence probing of biological systems. Depending on the separation between the donor (D) and the acceptor (A), the process can be described accurately by various theories accounting for the electromagnetic interaction between the two species. For the D-A distance range from about 2-6 $\mathrm{nm}$ which is most relevant for applications to many biological and photochemical studies, the wellestablished Förster theory ${ }^{1}$ based on the electrostatic dipoledipole interaction between $\mathrm{D}$ and $\mathrm{A}$ has been found very successful for the understanding of the energy transfer involved. For even closer distances, Dexter ${ }^{2}$ recognized that electronic exchange and multipolar interactions become important and a full quantum mechanical treatment must be implemented. On the other hand, in the far distance regime, full electrodynamics is needed to account for "radiative transfer" between D and A when retardation effects are important in such process. Our interest in the present work will focus on the so-called Förster resonance energy transfer (FRET) between D and A located in the vicinity of a metallic nanoparticle, in which case significant enhancement of the transfer process could be achieved via the plasmonic excitation of the surface electrons of the particle.

It is well known that while FRET is a very useful process which can be applied, for example, as a rule for spectroscopic measurements ${ }^{3}$; it is a rather weak process which goes down as inversed sixth power of the D-A separation with a transfer rate $\sim R^{-6}$. In fact, one can introduce a length scale known as the Förster distance $\left(R_{0}\right)$ at which FRET is $50 \%$ efficient, ${ }^{4}$ and it is found that $R_{0} \sim$ a few nanometers in most practical situations. Hence in order to extend the range of FRET in many spectroscopic application of the process, enhancement of the transfer rate is of prime importance and has been studied extensively in the literature. One wellestablished mechanism will be the utilization of the plasmonic resonant field from the collective oscillation of the surface electrons of a metallic structure.

Since the first discovery of the surface plasmon resonance enhancement of the Raman and many other spectroscopic processes in the vicinity of a metal structure more than three decades ago, this mechanism has also been applied to D-A energy transfer in recent years. For example, Andrews and Barnes have recently demonstrated convincingly enhanced FRET within a metallic cavity ${ }^{5}$; as well as enhanced radiative transfer across a relatively thick $(>100 \mathrm{~nm})$ metal film $^{6}$ via the coupling of the SP's of the metal to the near fields of the donor and acceptor molecules. Besides planar structures, localized surface plasmon (LSP) from metallic nanoparticles has also been applied to achieve enhancements of various D-A energy transfer processes in recent years. Using isolated metal particles, it was first shown theoretically ${ }^{7,8}$ that orders of magnitude enhancement can be obtained for FRET with the D-A pair located in close proximity of a spheroidal metal particle of sizes $\sim$ tens of nanometers. Both ensembleaveraged experiments ${ }^{9}$ and single-molecule experiments ${ }^{4,10}$ have been performed in recent years with positive results indicating that both the Förster distance and the FRET rate constant can be increased by several times using metallic particle enhancement techniques. In addition, nanoparticle chain has also been used in place of single metal particles with the D's and A's located at different particle sites along 
the chain. ${ }^{11}$ Coupling of LSP's across the particles along the chain then enables enhanced energy transfer between the D and the A over a large range of separations.

Besides FRET for molecules, plasmonic enhancements from metal particles have also been studied for possible facilitation of the transfer of optically excited excitonic energy between pairs of semiconductor quantum dots (QDs). ${ }^{12}$ Potential applications of QD labeling in place of fluorescing molecules in biological studies is anticipated due partly to the photobleaching problems with the latter labeling system. The recent theoretical work ${ }^{12}$ which goes beyond the dipole approximation for the D's and A's has revealed the complicated coupled dynamics between the excitons in the QD's and the SP's in the metal particle, implying a careful choice of excitation frequency must be made in order to optimize the enhancement of FRET in this process.

The purpose of our present work is to provide a more accurate description of the optical response of the metal particle in this enhanced FRET processes. While the previous works have established models based on ${ }^{1,2,7,8,11}$ and beyond ${ }^{12}$ the dipole approximation for the D's and the A's, the metal particle is all the time modeled according to its dielectric response within classical electrodynamics. For example, in one of the latest works that studied the nanoparticle-modified emission rates for a single admolecule, in which an improved quasistatic model was shown to agree well with the one from exact electrodynamics even for relatively large particle sizes; the dielectric response of the metallic sphere was still described simply using the Drude model. ${ }^{13}$ However, in future applications of FRET, it is reasonable to envision that nanoparticles of smaller sizes will be used to link to these D's and A's, achieving an increase in the flexibility and accuracy of using them to tag various biological samples to be probed. When these metal particles shrink down to below about 10 $\mathrm{nm}$, classical dielectric description of their response will be limited and possible quantum mechanical effects will start to emerge. While accounting for the full quantum mechanics of such a large number of free electrons in the nanoparticle is rather nontrivial, ${ }^{14}$ we shall in this work study these effects to some extent via a phenomenological approach using the formulations from nonlocal optics. ${ }^{15}$ Furthermore, besides for ultrasmall particles, these nonlocal effects will also become very significant when the D's and A's are placed in very close proximity to the particle surface, rendering the previous theories $1,2,7,8,11,12$ inaccurate since they are all based on a local response electromagnetic theory. To appreciate these "close-distance effects," we refer to a very recent investigation on the plasmonics of a system of interacting metallic nanoparticles (without admolecules) in which these nonlocal effects have been found rather significant when the particles are very close to each other. ${ }^{16}$

We shall give a brief summary of our formulation for a nonlocal FRET theory in the next section in which we shall consider both metallic solid particles and nanoshells as our plasmonic systems. This latter system has become a highly versatile plasmonic source in the last decade due to its tunability for the SP resonance via adjustment of the shell thickness and core material ${ }^{17}$; and possible enhanced FRET from such a nanoshell has just began to draw attentions from researchers very recently. In one of the latest works, Durach et

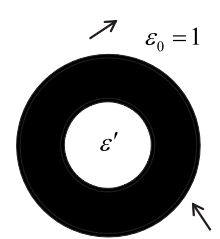

(a)

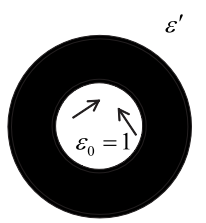

(b)

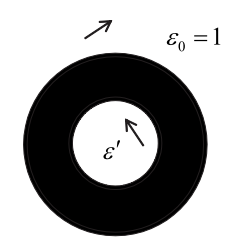

(c)
FIG. 1. The three different geometries for the dipoles D and the A near the nanoshell: (a) D and A are both outside the nanoshell; (b) both inside the nanoshell; and (c) D outside and A inside the nanoshell.

al. ${ }^{18}$ had studied theoretically FRET between two QD's (modeled as point dipoles) located, respectively, outside the two poles of a spherical nanoshell. They observed in general greater enhancements for FRET in the shell case compared to that in case with a solid metal particle, and many higher multipole resonances could emerge in the shell case. However, this previous work had focused only on shells of relatively larger sizes (outer radius $\sim 20 \mathrm{~nm}$ ) so that nonlocal effects were not studied. Also the D and A are studied only at directly opposite locations near the poles outside the nanoshell. Our formulation here will account for nonlocal optical response from the metal, and will allow the D and the A to be placed at arbitrary locations with arbitrary relative orientations outside the metallic structure as in Ref. 8; and in the nanoshell case, one (or both) of them can also be placed inside the cavity formed by the shell. In our numerical studies, we shall see that nonlocal effects will generally lead to lower enhancements at the low frequencies within each coupled mode than otherwise expected for the FRET process; and these effects are in particular prominent for shells of small dimensions.

\section{THEORY}

Let us consider two point dipoles in arbitrary locations and orientations with respect to a metallic nanoshell, with the dipoles (D and A) located either both outside the shell; both inside ; or one inside and one outside the cavity formed by the shell (Fig. 1). The third case will bear some similarity to the case studied by Andrews and Barnes with FRET taking place between $\mathrm{D}$ and $\mathrm{A}$ across a metal film in the planar geometry, ${ }^{6}$ while the same authors had also studied earlier the FRET process in a planar cavity ${ }^{5}$ which resembled our second case here in spherical geometry. Also, the first case will identify with the system treated by Gersten and Nitzan ${ }^{7}$ for a spherical metal particle in the limit with the inner radius $a \rightarrow 0$. In addition, since all the dimensions (nanoshell sizes, molecule-shell distances, and intermolecular distances) are assumed much smaller than the emission and absorption wavelengths, electrostatic solutions to the problem will be employed. ${ }^{7,8}$ Our interest here, as explained above, is focused on the possible nonlocal (could be quantal) effects from the metallic electrons which are significant only for small particle sizes and/or close molecule-particle distances.

To calculate the FRET rate between the D and the A, we follow the original theory ${ }^{1,7,8}$ which applied the Fermi 
golden rule and obtain the following expression:

$$
K(\omega)=\frac{9 c^{4}}{8 \pi} \frac{\sigma_{a}(\omega) \Gamma_{d}(\omega)}{\omega^{4}}|U(\omega)|^{2},
$$

where $\sigma_{a}(\omega)$ is the absorption cross-section of the acceptor, $\Gamma_{d}(\omega)$ is the emission rate per unit frequency of the donor, and $U(\omega)$ is the interaction energy between $\mathrm{D}$ and $\mathrm{A}$ in the presence of the metallic nanoparticle. ${ }^{7,8}$ Note that the resonance condition implies the transition frequency is the same for both $\mathrm{D}$ and $\mathrm{A}$. In principle, the presence of the nanoshell will also modify the absorption and emission of the dipoles. However, in the spirit of first-order perturbation theory (via the Fermi golden rule), unperturbed states of the molecules are used which lead to the conclusion that one merely needs to use the free molecular values for $\sigma_{a}(\omega)$ and $\Gamma_{d}(\omega)$ in Eq. (1). Thus in this standard approach, ${ }^{1,7,8}$ the presence of the metal particle will only modify $U(\omega)$ which can be solved from the appropriate boundary value problem involving the nanoshell and the two dipoles. We shall follow the nonlocal dielectric response theory developed by Fuchs and co-workers ${ }^{19,20}$ to calculate the effects on $U(\omega)$ for the three cases in Fig. 1.

\section{A. Case (a): Both dipoles outside the nanoshell}

For the geometry in Fig. 1(a), we divide the boundary problem into three zones: the region outside the nanoshell $(r>b)$ which is taken as vacuum, the dielectric core $(r<a)$ which is assumed to have real dielectric constant $\varepsilon^{\prime}$, and the metallic shell $(a<r<b)$ which is described by a nonlocal dielectric function $\varepsilon(k, \omega)$. For this case with both dipoles outside the shell, we follow the works of Fuchs et al. ${ }^{19,20}$ and consider the following general solution for the electrostatic potentials associated with the electric field $(\vec{E}=-\vec{\nabla} \Phi)$ and the displacement vector $\left(\vec{D}=-\vec{\nabla} \Phi_{D i s}\right)$, respectively,

$$
\begin{gathered}
\Phi(\vec{r})= \begin{cases}\sum_{\ell=0}^{\infty} \sum_{m=-\ell}^{\ell} A_{\ell m} r^{\ell} Y_{\ell m}(\theta, \phi), & r<a \\
\sum_{\ell=0}^{\infty} \sum_{m=-\ell}^{\ell}\left[\delta_{a} a^{2} F_{\ell}(a, r)+\delta_{b} b^{2} F_{\ell}(b, r)\right] Y_{\ell m}(\theta, \phi), & a<r<b \\
\sum_{\ell=0}^{\infty} \sum_{m=-\ell}^{\ell} B_{\ell m} \frac{1}{r^{\ell+1}} Y_{\ell m}(\theta, \phi)+\Phi_{D}+\Phi_{A}, & b<r,\end{cases} \\
\Phi_{D i s}(a<r<b)=\sum_{\ell=0}^{\infty} \sum_{m=-\ell}^{\ell} \frac{1}{2 \ell+1}\left(\frac{a^{\ell+2}}{r^{\ell+1}} \delta_{a}+\frac{r^{\ell}}{b^{\ell-1}} \delta_{b}\right) Y_{\ell m}(\theta, \phi),
\end{gathered}
$$

where

$$
F_{\ell}(x, y)=\frac{2}{\pi} \int_{0}^{\infty} \frac{j_{\ell}(k x) j_{\ell}(k y)}{\varepsilon(k, \omega)} d k
$$

In the above Eqs. (2)-(4), $\Phi_{D}\left(\Phi_{A}\right)$ is the potential due to the point dipole located at the donor (acceptor) position [see appendix for details], $Y_{\ell m}$ is the spherical harmonic and $j_{\ell}$ the spherical Bessel function; $\delta_{a, b}$ are the quantities introduced to make the radial components of the displacement vector $D_{r}$ discontinuous into a fictitious medium at the boundaries, ${ }^{19,20}$ which resembles the presence of a fictitious surface current to provide a mechanism for introducing the nonlocal response of the shell. To determine the various unknown coefficients in Eq. (2), we apply the appropriate boundary conditions ${ }^{19,20}$ at $r=a$ and $r$ $=b$ to obtain the following matrix equation:

$$
\left(\begin{array}{cccc}
a^{\ell} & 0 & -a^{2} F_{\ell}(a, a) & -b^{2} F_{\ell}(b, b) \\
\varepsilon^{\prime} \ell a^{\ell-1} & 0 & \frac{\ell+1}{2 \ell+1} & -\frac{\ell}{2 \ell+1} a^{\ell-1} b^{-\ell+1} \\
0 & -b^{-\ell-1} & a^{2} F_{\ell}(a, b) & b^{2} F_{\ell}(b, b) \\
0 & (\ell+1) b^{-\ell-2} & -\frac{\ell+1}{2 \ell+1} a^{\ell+2} b^{-\ell-2} & \frac{\ell}{2 \ell+1}
\end{array}\right)\left(\begin{array}{c}
A_{\ell m} \\
B_{\ell m} \\
\delta_{a} \\
\delta_{b}
\end{array}\right)=\left(\begin{array}{c}
0 \\
0 \\
\left.\sum_{j=D, A} \widetilde{\Phi}_{\ell m j}\right|_{r \rightarrow b} \\
\left.\frac{\partial}{\partial b} \sum_{j=D, A} \tilde{\Phi}_{\ell m j}\right|_{r \rightarrow b}
\end{array}\right),
$$


from which the four coefficients $A_{\ell m}, B_{\ell m}, \delta_{a}$, and $\delta_{b}$ can be solved in terms of the potentials and their radial derivatives. In particular, $B_{\ell m}$ can be obtained in the following form (see appendix for the detailed expressions of $\Phi_{\ell m j}$ and $\widetilde{\Phi}_{\ell m j}$ ):

$$
B_{\ell m}=\left.\frac{\ell \gamma-b \beta}{(\ell+1) \gamma+b \beta} b^{\ell+1} \sum_{j=D, A} \tilde{\Phi}_{\ell m j}\right|_{r \rightarrow b},
$$

where

$$
\begin{aligned}
\gamma= & F_{\ell}(a, b) \ell a^{\ell+1} b^{-\ell+1}-a F_{\ell}(a, b) \varepsilon^{\prime} \ell(2 \ell+1) b^{2} F_{\ell}(b, a) \\
& +b^{2} F_{\ell}(b, b) \varepsilon^{\prime} \ell(2 \ell+1) a F_{\ell}(a, a)+(\ell+1) b^{2} F_{\ell}(b, b)
\end{aligned}
$$

and

$$
\begin{aligned}
\beta= & -\frac{\ell(\ell+1)}{2 \ell+1} a^{2 \ell+1} b^{-2 \ell-1}+(\ell+1) \ell \varepsilon^{\prime} a^{\ell+1} b^{-\ell} F_{\ell}(b, a) \\
& +\ell^{2} \varepsilon^{\prime} a F_{\ell}(a, a)+\frac{\ell(\ell+1)}{2 \ell+1} .
\end{aligned}
$$

From this, we can then calculate the interaction energy between the donor and the acceptor, e.g., $U=-\left.\vec{\mu}_{D} \cdot \vec{E}\right|_{\vec{r}=\vec{r}_{D}}$, where $\vec{E}$ is the total electric field produced by the acceptor and the metal particle at the donor position. The final result can be expressed as follows:

$$
\begin{aligned}
U= & -\mu_{r_{D}} \sum_{\ell=0}^{\infty} \sum_{m=-\ell}^{\ell}\left[B_{\ell m}^{\prime} \frac{\ell+1}{r_{D}^{\ell+2}} Y_{\ell m}\left(\theta_{D}, \phi_{D}\right)-\left.\frac{\partial}{\partial r_{D}} \Phi_{\ell m A}\right|_{r \rightarrow r_{D}}\right] \\
& +\mu_{\theta_{D}} \sum_{\ell=0}^{\infty} \sum_{m=-\ell}^{\ell}\left[B_{\ell m}^{\prime} \frac{1}{r_{D}^{\ell+2}} \frac{\partial Y_{\ell m}\left(\theta_{D}, \phi_{D}\right)}{\partial \theta_{D}}+\left.\frac{\partial}{\partial \theta_{D}} \Phi_{\ell m A}\right|_{r \rightarrow r_{D}}\right] \\
& +\frac{\mu_{\phi_{D}}}{\sin \theta_{D}} \sum_{\ell=0}^{\infty} \sum_{m=-\ell}^{\ell}\left[B_{\ell m}^{\prime} \frac{1}{r_{D}^{\ell+2}} \frac{\partial Y_{\ell m}\left(\theta_{D}, \phi_{D}\right)}{\partial \phi_{D}}\right. \\
& \left.+\left.\frac{\partial}{\partial \phi_{D}} \Phi_{\ell m A}\right|_{r \rightarrow r_{D}}\right]
\end{aligned}
$$

where $B_{\ell m}^{\prime}$ is as given in Eq. (6) with the donor term $\widetilde{\Phi}_{\ell m D}$ removed from the sum. Hence the FRET enhancement $|A(\omega)|^{2}=\left|\frac{U}{U_{0}}\right|^{2}$ can be calculated, with $U_{0}$ being the electrostatic energy between the donor and the acceptor in free space. It is clear from the above results that our formulation here can allow for arbitrary location $\left(r_{j}, \theta_{j}, \phi_{j}\right)$ for the dipoles with arbitrary orientation which determines the dipole moment components $\left(\mu_{r_{j}}, \mu_{\theta_{j}}, \mu_{\phi_{j}}\right)$.

\section{B. Case (b): Both dipoles inside the nanoshell}

For the case in Fig. 1(b) where we have both donors and acceptors inside the vacuum enclosed by the shell, and the outside medium with a real dielectric constant $\varepsilon^{\prime}$, we can formulate the boundary value problem in a way very similar to the above case (a). Thus we have

$$
\begin{gathered}
\Phi(\vec{r})= \begin{cases}\sum_{\ell=0}^{\infty} \sum_{m=-\ell}^{\ell} C_{\ell m} r^{\ell} Y_{\ell m}(\theta, \phi)+\Phi_{D}+\Phi_{A}, & r<a \\
\sum_{\ell=0}^{\infty} \sum_{m=-\ell}^{\ell}\left[\delta_{a} a^{2} F_{\ell}(a, r)+\delta_{b} b^{2} F_{\ell}(b, r)\right] Y_{\ell m}(\theta, \phi), & a<r<b \\
\sum_{\ell=0}^{\infty} \sum_{m=-\ell}^{\ell} D_{\ell m} \frac{1}{r^{\ell+1}} Y_{\ell m}(\theta, \phi), & r>b,\end{cases} \\
\Phi_{D i s}(a<r<b)=\sum_{\ell=0}^{\infty} \sum_{m=-\ell}^{\ell} \frac{1}{2 \ell+1}\left(\frac{a^{\ell+2}}{r^{\ell+1}} \delta_{a}+\frac{r^{\ell}}{b^{\ell-1}} \delta_{b}\right) Y_{\ell m}(\theta, \phi),
\end{gathered}
$$

and the corresponding matrix equation for the four unknown coefficients

$$
\left(\begin{array}{cccc}
a^{\ell} & 0 & -a^{2} F_{\ell}(a, a) & -b^{2} F_{\ell}(b, a) \\
\ell a^{\ell-1} & 0 & \frac{\ell+1}{2 \ell+1} & -\frac{\ell}{2 \ell+1} a^{\ell-1} b^{-\ell+1} \\
0 & -b^{-\ell-1} & a^{2} F_{\ell}(a, b) & b^{2} F_{\ell}(b, b) \\
0 & \varepsilon^{\prime}(\ell+1) b^{-\ell-2} & -\frac{\ell+1}{2 \ell+1} a^{\ell+2} b^{-\ell-2} & \frac{\ell}{2 \ell+1}
\end{array}\right)\left(\begin{array}{c}
C_{\ell m} \\
D_{\ell m} \\
\delta_{a} \\
\delta_{b}
\end{array}\right)=\left(\begin{array}{c}
-\left.\sum_{j=D, A} \widetilde{\Phi}_{\ell m j}\right|_{r \rightarrow a} \\
-\left.\frac{\partial}{\partial a} \sum_{j=D, A} \tilde{\Phi}_{\ell m j}\right|_{r \rightarrow a} \\
0 \\
0
\end{array}\right) .
$$


Solving Eq. (12), we obtain

$$
C_{\ell m}=\left.\frac{(\ell+1) \tilde{\gamma}+a \tilde{\beta}}{\ell \tilde{\gamma}-a \tilde{\beta}} \frac{1}{a^{\ell}} \sum_{j=D, A} \widetilde{\Phi}_{\ell m j}\right|_{r \rightarrow a},
$$

where

$$
\begin{aligned}
\widetilde{\gamma}= & -F_{\ell}(b, a)(\ell+1) a^{\ell+2} b^{-\ell}+b F_{\ell}(a, b) \varepsilon^{\prime}(\ell+1)(2 \ell \\
& +1) a^{2} F_{\ell}(b, a)-b F_{\ell}(b, b) \varepsilon^{\prime}(\ell+1)(2 \ell+1) a^{2} F_{\ell}(a, a) \\
& -\ell a^{2} F_{\ell}(a, a)
\end{aligned}
$$

and

$$
\begin{aligned}
\tilde{\beta}= & -\frac{\ell(\ell+1)}{2 \ell+1} a^{2 \ell+1} b^{-2 \ell-1}+(\ell+1) \ell \varepsilon^{\prime} a^{\ell+1} b^{-\ell} F_{\ell}(a, b)+(\ell \\
& +1)^{2} \varepsilon^{\prime} b F_{\ell}(b, b)+\frac{\ell(\ell+1)}{2 \ell+1} .
\end{aligned}
$$

In a similar way, we obtain the energy between the donor and the acceptor as follows:

$$
\begin{aligned}
U= & \mu_{r_{D}} \sum_{\ell=0}^{\infty} \sum_{m=-\ell}^{\ell}\left[C_{\ell m}^{\prime} \ell r_{D}^{\ell-1} Y_{\ell m}\left(\theta_{D}, \phi_{D}\right)+\left.\frac{\partial}{\partial r_{D}} \Phi_{\ell m A}\right|_{r \rightarrow r_{D}}\right] \\
& +\mu_{\theta_{D}} \sum_{\ell=0}^{\infty} \sum_{m=-\ell}^{\ell}\left[C_{\ell m}^{\prime} r_{D}^{\ell-1} \frac{\partial Y_{\ell m}\left(\theta_{D}, \phi_{D}\right)}{\partial \theta_{D}}+\left.\frac{\partial}{\partial \theta_{D}} \Phi_{\ell m A}\right|_{r \rightarrow r_{D}}\right] \\
& +\frac{\mu_{\phi_{D}}}{\sin \theta_{D}} \sum_{\ell=0}^{\infty} \sum_{m=-\ell}^{\ell}\left[C_{\ell m}^{\prime} r_{D}^{\ell-1} \frac{\partial Y_{\ell m}\left(\theta_{D}, \phi_{D}\right)}{\partial \phi_{D}}\right. \\
& \left.+\left.\frac{\partial}{\partial \phi_{D}} \Phi_{\ell m A}\right|_{r \rightarrow r_{D}}\right],
\end{aligned}
$$

where $C_{\ell m}^{\prime}$ is as given in Eq. (13) with the donor term $\widetilde{\Phi}_{\ell m D}$ removed from the sum. Again, $|A(\omega)|^{2}$ can be calculated from Eq. (16) as before.

\section{Case (c): One dipole inside and the other outside the nanoshell}

Finally, we consider the configuration of Fig. 1(c) where the two dipoles are separated by the metal shell with one dipole inside (dielectric constant $\varepsilon^{\prime}$ ) and one outside (vacuum) the shell. The form of the general solution is thus given by

$$
\begin{gathered}
\Phi(\vec{r})= \begin{cases}\sum_{\ell=0}^{\infty} \sum_{m=-\ell}^{\ell} E_{\ell m} r^{\ell} Y_{\ell m}(\theta, \phi)+\Phi_{A}, & r<a \\
\sum_{\ell=0}^{\infty} \sum_{m=-\ell}^{\ell}\left[\delta_{a} a^{2} F_{\ell}(a, r)+\delta_{b} b^{2} F_{\ell}(b, r)\right] Y_{\ell m}(\theta, \phi), & a<r<b \\
\sum_{\ell=0}^{\infty} \sum_{m=-\ell}^{\ell} G_{\ell m} \frac{1}{r^{\ell+1}} Y_{\ell m}(\theta, \phi)+\Phi_{D}, & b<r,\end{cases} \\
\Phi_{D i s}(a<r<b)=\sum_{\ell=0}^{\infty} \sum_{m=-\ell}^{\ell} \frac{1}{2 \ell+1}\left(\frac{a^{\ell+2}}{r^{\ell+1}} \delta_{a}+\frac{r^{\ell}}{b^{\ell-1}} \delta_{b}\right) Y_{\ell m}(\theta, \phi) .
\end{gathered}
$$

Using again the appropriate boundary conditions we obtain the following matrix equation for the unknown coefficients:

$$
\left(\begin{array}{cccc}
a^{\ell} & 0 & -a^{2} F_{\ell}(a, a) & -b^{2} F_{\ell}(b, b) \\
\varepsilon^{\prime} \ell a^{\ell-1} & 0 & \frac{\ell+1}{2 \ell+1} & -\frac{\ell}{2 \ell+1} a^{\ell-1} b^{-\ell+1} \\
0 & -b^{-\ell-1} & a^{2} F_{\ell}(a, b) & b^{2} F_{\ell}(b, b) \\
0 & (\ell+1) b^{-\ell-2} & -\frac{\ell+1}{2 \ell+1} a^{\ell+2} b^{-\ell-2} & \frac{\ell}{2 \ell+1}
\end{array}\right)\left(\begin{array}{c}
E_{\ell m} \\
G_{\ell m} \\
\delta_{a} \\
\delta_{b}
\end{array}\right)=\left(\begin{array}{c}
-\left.\widetilde{\Phi}_{\ell m A}\right|_{r \rightarrow a} \\
-\left.\frac{\partial}{\partial a} \widetilde{\Phi}_{\ell m A}\right|_{r \rightarrow a} \\
\left.\widetilde{\Phi}_{\ell m D}\right|_{r \rightarrow b} \\
\left.\frac{\partial}{\partial b} \widetilde{\Phi}_{\ell m D}\right|_{r \rightarrow b}
\end{array}\right)
$$

From Eq. (19), we obtain $G_{\ell m}$ in the following more complicated form:

$$
G_{\ell m}=\frac{\left.\alpha_{1} \tilde{\Phi}_{\ell m A}\right|_{r \rightarrow a}-\left.\alpha_{2} \frac{\partial}{\partial a} \widetilde{\Phi}_{\ell m A}\right|_{r \rightarrow a}-\left.\alpha_{3} \widetilde{\Phi}_{\ell m D}\right|_{r \rightarrow b}+\left.\alpha_{4} \frac{\partial}{\partial b} \widetilde{\Phi}_{\ell m D}\right|_{r \rightarrow b}}{\alpha_{3} b^{-\ell-1}+\alpha_{4}(\ell+1) b^{-\ell-2}},
$$


where $\alpha_{1}, \alpha_{2}, \alpha_{3}$, and $\alpha_{4}$ are given as follows:

$$
\begin{gathered}
\alpha_{1}=\varepsilon^{\prime} a^{\ell+1} \frac{\ell^{2}}{2 \ell+1} F_{\ell}(a, b)+\varepsilon^{\prime} \frac{\ell(\ell+1)}{2 \ell+1} a^{2 \ell+1} b^{-\ell} F_{\ell}(b, b), \\
\alpha_{2}=\frac{\ell}{2 \ell+1} a^{\ell+2} F_{\ell}(a, b)+\frac{\ell+1}{2 \ell+1} a^{2 \ell+2} b^{-\ell} F_{\ell}(b, b), \\
\alpha_{3}=\frac{\ell(\ell+1)}{(2 \ell+1)^{2}} a^{\ell}+\frac{\ell(\ell+1)}{2 \ell+1} \varepsilon^{\prime} a^{2 \ell+1} b^{-\ell} F_{\ell}(b, a) \\
+\frac{\ell^{2}}{2 \ell+1} a^{\ell+1} \varepsilon^{\prime} F_{\ell}(a, a)-\frac{\ell(\ell+1)}{(2 \ell+1)^{2}} a^{3 \ell+1} b^{-2 \ell-1}, \\
\alpha_{4}=\frac{\ell+1}{2 \ell+1} a^{\ell} b^{2} F_{\ell}(b, b)-F_{\ell}(b, a) F_{\ell}(a, b) b^{2} a^{\ell+1} \ell \varepsilon^{\prime} \\
+F_{\ell}(a, a) F_{\ell}(b, b) a^{\ell+1} b^{2} \varepsilon^{\prime} \ell+\frac{\ell}{2 \ell+1} F_{\ell}(a, b) a^{2 \ell+1} b^{-\ell+1}
\end{gathered} .
$$

We hence derive the energy between the donor and the acceptor in this case as follows:

$$
\begin{aligned}
U= & -\mu_{r_{D}} \sum_{\ell=0}^{\infty} \sum_{m=-\ell}^{\ell} G_{\ell m}^{\prime}(\ell+1) \frac{1}{r_{D}^{\ell+2}} Y_{\ell m}\left(\theta_{D}, \phi_{D}\right) \\
& +\mu_{\theta_{D}} \sum_{\ell=0}^{\infty} \sum_{m=-\ell}^{\ell} G_{\ell m}^{\prime} \frac{1}{r_{D}^{\ell+2}} \frac{\partial Y_{\ell m}\left(\theta_{D}, \phi_{D}\right)}{\partial \theta_{D}} \\
& +\frac{\mu_{\phi_{D}}}{\sin \theta_{D}} \sum_{\ell=0}^{\infty} \sum_{m=-\ell}^{\ell} G_{\ell m}^{\prime} \frac{1}{r_{D}^{\ell+2}} \frac{\partial Y_{\ell m}\left(\theta_{D}, \phi_{D}\right)}{\partial \phi_{D}},
\end{aligned}
$$

where $G_{\ell m}^{\prime}$ is as given in Eq. (20) with the two terms involving $\widetilde{\Phi}_{\ell m D}$ removed, and hence $|A(\omega)|^{2}$ can be obtained.

\section{NUMERICAL RESULTS AND DISCUSSION}

In all computations in this section, we shall assume the dielectric as a certain kind of polymer with $\varepsilon^{\prime}=1.5$, and consider silver as the metal described by either the Drude (local) or the hydrodynamic (nonlocal) model with the latter expressible in the following form ${ }^{21}$ :

$$
\varepsilon(k, \omega)=1-\frac{\omega_{p}^{2}}{\omega(\omega+i \Gamma)-v_{0}^{2} k^{2}},
$$

where $\omega_{p}$ and $\Gamma$ are the plasmon frequency and damping factor of the electrons, respectively, and $v_{0}=\sqrt{\frac{3}{5}} v_{F}$ with $v_{F}$ being the Fermi velocity and the Drude model is obtained simply by setting $k=0$ in Eq. (23). In this case, the function in Eq. (4) can be obtained in close form as follows ${ }^{21}$ :

$$
\begin{aligned}
F_{\ell}(x, y)= & \frac{1}{\varepsilon(2 \ell+1)}\left(\frac{x}{y}\right)^{\ell+1 / 2} \frac{1}{\sqrt{x y}} \\
& +\frac{1}{\sqrt{x y}}\left(\frac{\omega_{p}^{2}}{\omega_{p}^{2}-\omega(\omega+i \Gamma)}\right) I_{\ell+1 / 2}(\bar{\kappa} x) K_{\ell+1 / 2}(\bar{\kappa} y),
\end{aligned}
$$

where $x<y, \varepsilon=\varepsilon(0, \omega)$ is the Drude function, $I_{\nu}$ and $K_{\nu}$ are

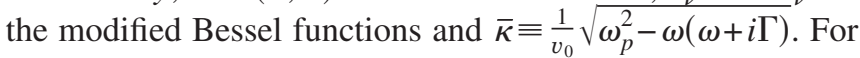
silver, the plasmon frequency is taken as $\omega_{p}=1.36$ $\times 10^{16} \mathrm{~s}^{-1}$, and the damping constant $\Gamma=\Gamma_{B U L K}+\frac{\alpha v_{F}}{b-a}$ is adopted to include the interface scattering term with $\Gamma_{B U L K}$ $=2.56 \times 10^{13} \mathrm{~s}^{-1}$. Without loss of generality, we shall set the geometric factor $\alpha=1$, in accord with recent simulation studies on these nanoshells. ${ }^{22}$

To illustrate the nonlocal optical effects discussed above, we first study numerically FRET enhancement from a solid metal particle of $3 \mathrm{~nm}$ radius. To demonstrate our formulation is indeed capable of accounting for any arbitrary location and orientation of the dipoles, Fig. 2 shows the factor $|U(\omega)|^{2}$ in Eq. (1) as a function of the relative positions and orientations of the dipoles D and A; with Fig. 2(a) varying in the position of A which is in parallel alignment with D; and 2(b) at fixed positions but with the orientation varied. It is of interest to note the following features: (i) the nonlocal effect leads to smaller enhanced FRET in general (for $\theta>90^{\circ}$ when $\mathrm{D}$ and A are relatively far apart) and such effect decreases as the angular separation between the D and A decreases due to the dominance of direct energy transfer, this dominance, interplaying with the particle-induced effects, can in fact yield an overall greater enhancement for a range of close D-A distances; (ii) the "null transfer condition" [i.e., relative position between D and A at which $\left.|U(\omega)|^{2}=0\right]$ is slightly modified due to the presence of the nanoparticle; and (iii) the orientation effect depends very much on the relative locations of the dipoles [Fig. 2(b)]; and when $\mathrm{A}$ is located within the lower half of the metal sphere, we estimated that the enhancement $|A(\omega)|^{2}$ is rather insensitive to the orientation of the dipoles. ${ }^{8}$ It is worthy to note that for two aligned molecular dipoles as in Fig. 2(a), enhancement from the metal particle is guaranteed only when D and A are a little farther from each other with A lying within the lower hemisphere of the particle.

To switch to metallic nanoshells, Fig. 3 shows the FRET enhancement spectra as a function of transition frequency for the three configurations in Fig. 1 for a 8/10 (i.e., $a=8 \mathrm{~nm}$ and $b=10 \mathrm{~nm}$ ) nanoshell. For simplicity, the D and A are located along the $z$ axis at the north and south poles, respectively, at close distances from either the inner or the outer shell surface. From these it is worthy to note: (i) the enhanced FRET centered around the hybridized bonding (lowfrequency) and antibonding (high-frequency) plasmonic modes; (ii) the appearance of several lowest order multipole bonding modes (starting at $\omega \sim 0.35 \omega_{p}$ for the dipole mode) with stronger enhancements for the higher order ones; (iii) the slight diminution of the enhancement at the lowfrequency portion of each coupled mode due to nonlocal effects; (iv) the slight blueshifted resonance peaks due to 

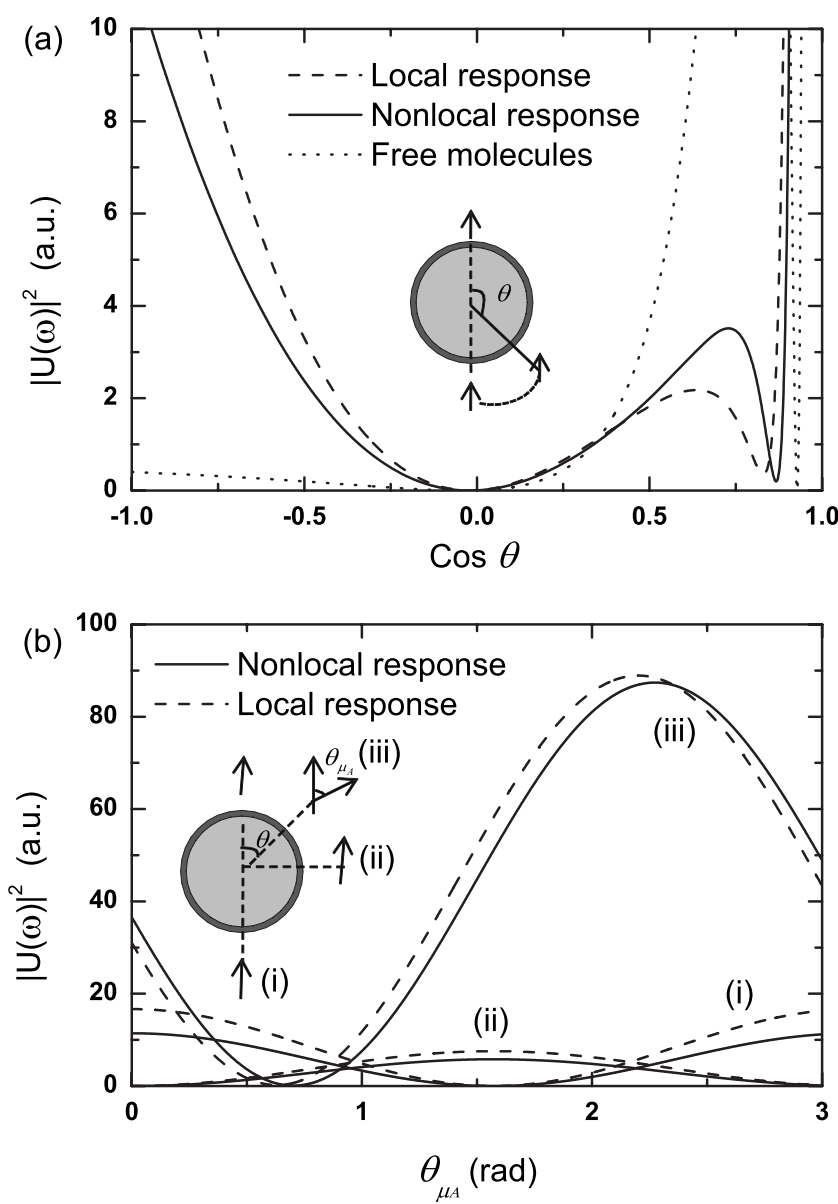

FIG. 2. Plot of the interaction energy factor $|U(\omega)|^{2}$ at a fixed frequency $\omega=6 \times 10^{15} \mathrm{rad} / \mathrm{s}$ for $\mathrm{D}$ and A outside a solid silver particle of $3 \mathrm{~nm}$ radius, with the radial coordinates for $\mathrm{D}$ and $\mathrm{A}$ fixed at $r_{D}=4 \mathrm{~nm}$ and $r_{A}=6 \mathrm{~nm}$ : (a) with orientations of D and A aligned along the $z$ direction, D fixed on the $z$ axis and A varied in its angular coordinate $\theta$; and (b) with the orientation of A, $\theta_{\mu_{A}}$ varied while its location fixed at (i) $\theta=\pi$, (ii) $\theta=\frac{\pi}{2}$ and (iii) $\theta=\frac{\pi}{4}$. Dash line: results from local dielectric response; solid line: results from nonlocal modeling; and dotted line: results from the free molecules (i.e., in the absence of metal particle).

these effects; and (v) the relatively much lower enhancement factor attainable for the case with both $\mathrm{D}$ and $\mathrm{A}$ inside the shell due to the large value of $U_{0}$ at such close distance between the molecules. Note that the different multipolar antibonding modes are so close and cannot be differentiated in our plots (they can be checked by plotting each individual multipole mode separately). It is a little surprise to point out that these antibonding modes were not observed in a previous study of similar FRET process [for case 3(a)] near a metallic nanoshell. ${ }^{18}$ It is also of interest to note that while the bonding modes provide greater enhancements in general than the antibonding modes in cases 3(a) and 3(c) ; the situation can be reversed when both dipoles are within the shell [case 3(b)]. This happens because our cases 3(a) and 3(b) are just reciprocal to each other and hence the cavity modes are for sure higher in frequency than the modes of the outer surface. Since the dipoles in 3(b)interact strongly with these cavity modes, a greater enhancement is achieved at the high-

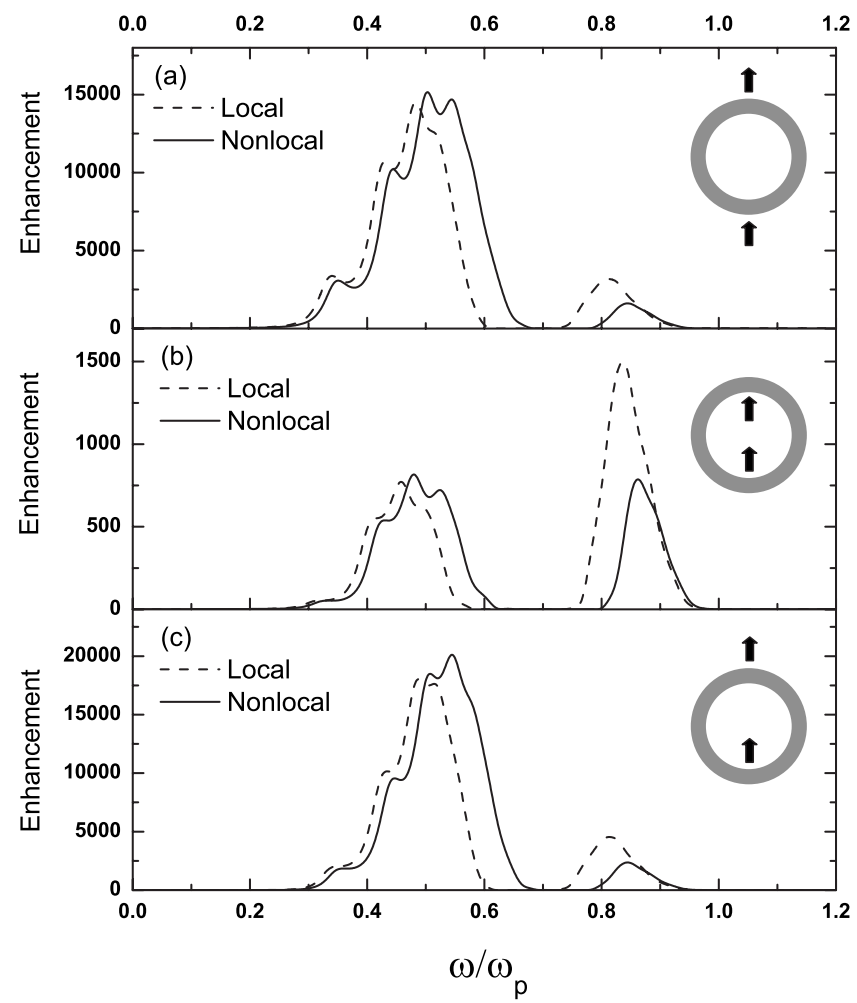

FIG. 3. Enhancement $|A(\omega)|^{2}$ as a function of transition frequency for the three configurations in Fig. 1 with $a=8 \mathrm{~nm}$ and $b$ $=10 \mathrm{~nm}$ : (a) both D and A outside the nanoshell $\left(r_{D}=10.5 \mathrm{~nm}\right.$ and $r_{A}=12 \mathrm{~nm}$ ); (b) both D and A inside $\left(r_{D}=7.5 \mathrm{~nm}\right.$ and $\left.r_{A}=6 \mathrm{~nm}\right)$ and (c) D outside and $\mathrm{A}$ inside the nanoshell $\left(r_{D}=11 \mathrm{~nm}\right.$ and $r_{A}$ $=7 \mathrm{~nm})$.

frequency antibonding modes. In passing, we also note that the bulk modes are not revealed in this calculation even for the nonlocal model. This is because of the exclusive dependence of the present FRET process on the enhanced surface fields due to the coupled plasmonic resonances. In addition, the large damping rate and the small bulk volume of the nanoshell also make these bulk resonances very insignificant. Similar phenomenon has been observed in our recent study of the nanoshell-enhanced SERS process. ${ }^{23}$

Figure 4 shows the "distance $(d)$ dependence" of the nanoshell-enhanced FRET rate at two fixed transition frequencies for the three configurations in Fig. 3. Note that we always have $\mathrm{D}$ located in the upper hemisphere and $\mathrm{A}$ in the lower hemisphere in this figure with positions as specified in the figure caption. Note also that in Figs. 4(a) and 4(b), the relative separation between $\mathrm{D}$ and $\mathrm{A}$ varies as $d$ changes and hence both direct and induced FRET rates change and interplay with each other at a given value of $d$. In the case of 4(c), this separation is fixed and hence the direct rate stays constant as $d$ varies. One can conclude with the following observations from Figs. 4(a)-4(c): (i) the nonlocal effects are most dramatic when both $\mathrm{D}$ and $\mathrm{A}$ are at close distances to the nanoshell surfaces, yielding lower enhancements at low transition frequency $\left(\omega=0.4 \omega_{p}\right)$ and higher enhancements at higher frequencies $\left(\omega=0.5 \omega_{p}\right)$ and (ii) such effects are significant only when the molecules D and A are located within a few nanometer distances from the nanoshell surfaces. 


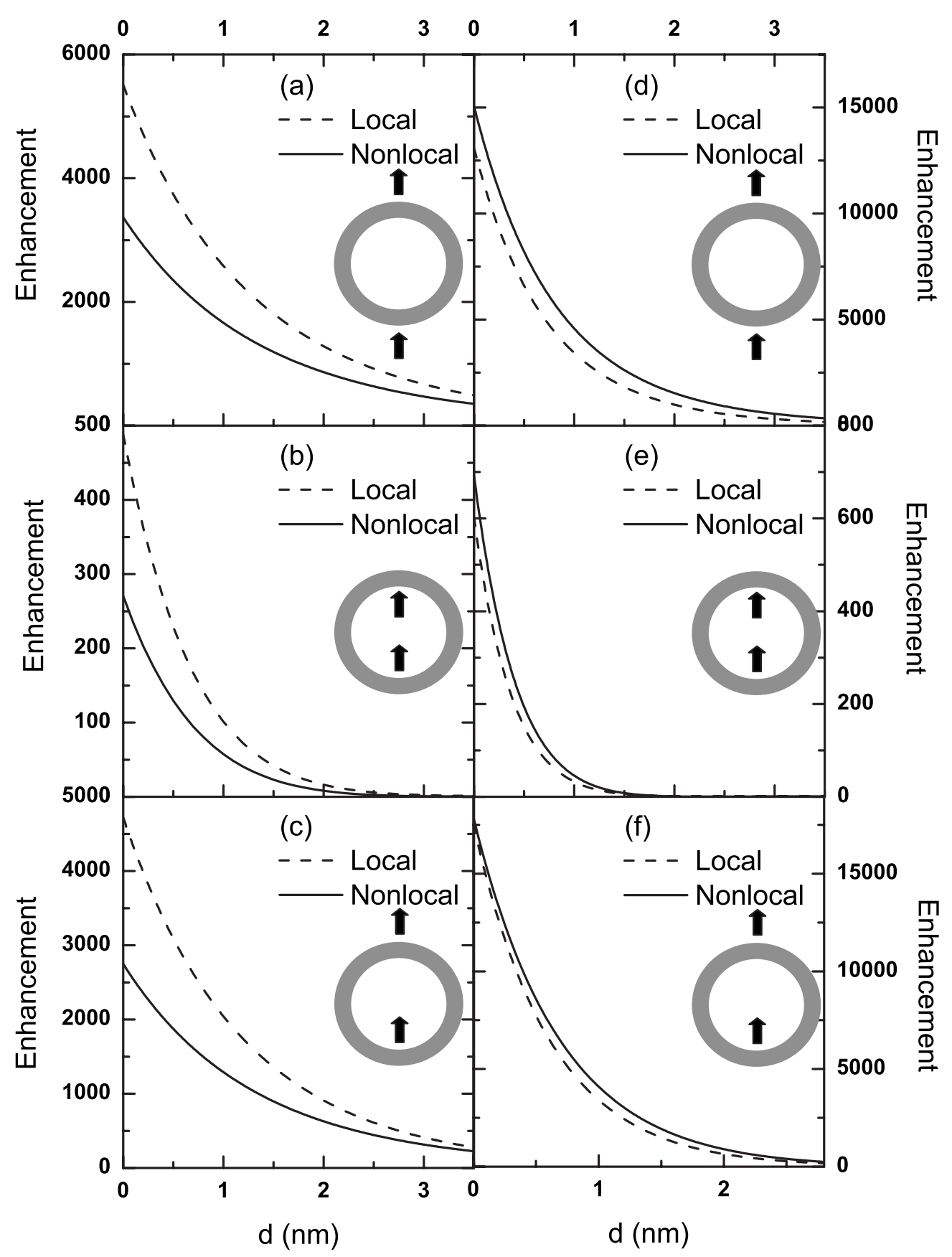

FIG. 4. Distance $(d)$ dependence of $|A(\omega)|^{2}$ at a fixed frequency $\omega=0.4 \omega_{p}$ for (a), (b), and (c); and $\omega=0.5 \omega_{p}$ for (d), (e), and (f). With the three geometries as in Fig. 3: (a) and (d) for both D and A outside $\left(r_{D}=10.5 \mathrm{~nm}+d\right.$ and $\left.r_{A}=12 \mathrm{~nm}+d\right)$; (b) and (e) for both D and A inside $\left(r_{D}=7.5 \mathrm{~nm}-d\right.$ and $\left.r_{A}=6 \mathrm{~nm}-d\right)$; (c) and (f) for D outside and A inside the nanoshell $\left(r_{D}=11 \mathrm{~nm}+d\right.$ and $\left.r_{A}=7 \mathrm{~nm}-d\right)$.

Figure 5 illustrates the dependence of the nonlocal effects on the thickness of the nanoshell, where we have considered only the case with one dipole outside and one inside the nanoshell. Figure 5(a) shows the FRET spectrum for a 8/10 nanoshell while Fig. 5(b) shows the same for a $6 / 10$ shell with the D and A located at the same fixed positions in both cases. With one of them (A) just below the center, the relative dominance of the dipole interaction is evident. From these two figures, one can observe the greater splitting between the coupled modes for the thinner nanoshell, as well as the more pronounced multipole interactions for the thicker shell. However, the greater enhancement observed for thin- ner shells in the previous work ${ }^{18}$ is not seen here. The main difference between our modeling and the one in Ref. 18 is that we have one of the $\mathrm{D}$ and A located inside the shell. This latter configuration will result in a closer distance between the molecule and the (inner) surface when the shell thickness increases, thus leading to an overall greater surface induced enhancement for the FRET rate. In addition, the more pronounced nonlocal effects expected for the thinner shell are not revealed in this calculation. This is likely due to the large surface damping rate $\left(\frac{\alpha v_{F}}{b-a}\right)$ for this thinner shell which has to some extent suppressed the nonlocal effects. This large 


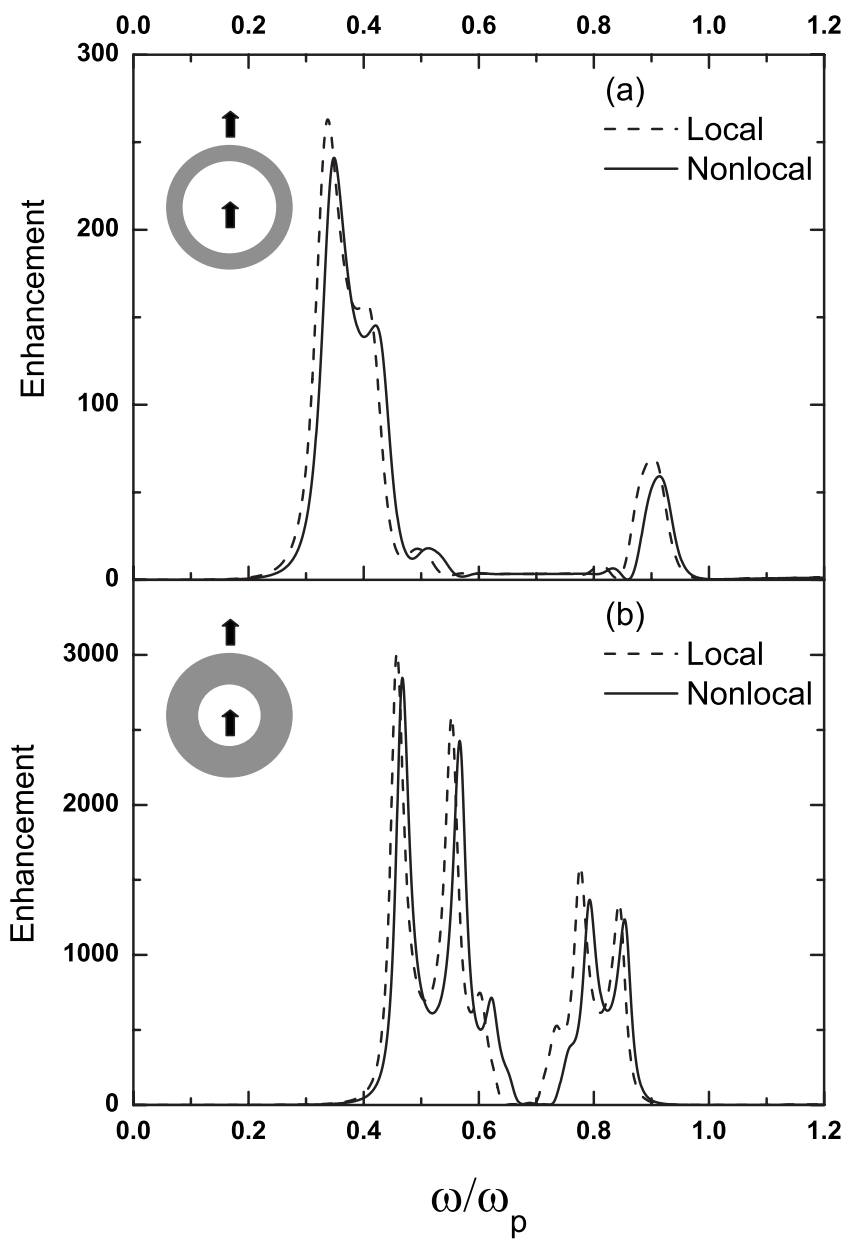

FIG. 5. Dependence of $|A(\omega)|^{2}$ on nanoshell thickness with molecular coordinates fixed on the $z$ axis at $r_{A}=2 \mathrm{~nm}$ and $r_{D}$ $=11 \mathrm{~nm}$. Dimensions for the nanoshell are $a=8 \mathrm{~nm}$ and $b$ $=10 \mathrm{~nm}$ for (a), and $a=6 \mathrm{~nm}$ and $b=10 \mathrm{~nm}$ for (b).

damping for the thinner shell is also responsible to the small enhancement observed in Fig. 5(a).

\section{CONCLUSION}

In this work, we have presented a study of the possible nonclassical effects from the metallic nanoparticle on the plasmonic enhanced FRET rates within the formulation of nonlocal optics. We have observed, in consistency with previous studies of these nonlocal effects on other optical/ spectroscopic processes, ${ }^{19-21,23}$ that the enhancements at low transition frequencies are in general not as high as predicted by classical electromagnetic (local) theory and the plasmonic resonances are in general blueshifted as the nanoparticle shrinks in its size. In addition, we have shown how it is possible to achieve enhanced FRET at much lower or higher frequencies by exploiting the hybridized plasmonic modes of the nanoshell, via the manipulation of the various parameters of the shell as well as the location of the donor and acceptor molecules. Our results for the three configurations of molecular locations demonstrate possible enhanced FRET for all the three cases, which is consistent with previous obser- vation for FRET enhancement from metal particles, ${ }^{4,8-10}$ as well as from planar metallic cavities ${ }^{5}$ and films. ${ }^{6}$ Our model, like all the previous ones, ${ }^{19-21,23}$ are limited by the semiclassical infinite barrier (SCIB) approximation which, while introducing the nonlocal response that provides a channel for quantum-mechanical dielectric response of the metal to be introduced, still does not guarantee the smooth continuity of the charge and field distributions across the geometric boundaries of the nanoparticle. In order to bring this model closer to the microscopic quantum description of the nanoparticle, it will be worth to extend the SCIB to account for smooth charge density profile across the boundary surfaces ${ }^{24}$ and investigate how the FRET rates are affected within such an improved nonlocal description of the metal particle. On the experimental side, it will be of great interest and challenge for the recent single-molecule FRET experiments ${ }^{4,10}$ to be extended to studies with nanoparticles of ultrasmall sizes $(<10 \mathrm{~nm}$, a regime already probed for novel effects on induced molecular fluorescence ${ }^{25}$ ), as well as studies with metallic nanoshells as the plasmonic source as modeled in our present work and in other recent theoretical study of the phenomenon. ${ }^{18}$

\section{ACKNOWLEDGMENTS}

We thank Railing Chang at the National Taiwan Ocean University for very useful discussions; and are grateful for the research support from the National Science Council of Taiwan, R.O.C., under Projects No. NSC-98-2120-M-002004, No. NSC-96-2923-M-002-002-MY3, and No. NSC 972811-M-002-018, respectively. We also acknowledge the support from National Taiwan University and National Center for Theoretical Sciences, Taipei Office. P. T. Leung would like to thank the additional support from a Fulbright Scholarship.

\section{APPENDIX}

Here we give details for the spherical function expansions of the potential $\Phi_{D}\left(\Phi_{A}\right)$ due to the point dipole located at the donor (acceptor) position.

$$
\begin{aligned}
\Phi_{j}(\vec{r}) \equiv & \sum_{\ell=0}^{\infty} \sum_{m=-\ell}^{\ell} \Phi_{\ell m j}(\vec{r}) \\
= & \mu_{r_{j}} \sum_{\ell=0}^{\infty} \sum_{m=-\ell}^{\ell} \frac{\partial}{\partial r_{j}}\left(\frac{r_{<}^{\ell}}{r_{>}^{\ell+1}}\right) \frac{(\ell-m) !}{(\ell+m) !} P_{\ell}^{m}(\cos \theta) \\
& \times P_{\ell}^{m}\left(\cos \theta_{j}\right) e^{i m\left(\phi-\phi_{j}\right)} \\
& -\frac{\mu_{\theta_{j}}}{r_{j}} \sum_{\ell=0}^{\infty} \sum_{m=-\ell}^{\ell} \frac{r_{<}^{\ell}}{r_{>}^{\ell+1}} \frac{(\ell-m) !}{(\ell+m) !} P_{\ell}^{m}(\cos \theta) \\
& \times\left[P_{\ell}^{m}\left(\cos \theta_{j}\right)\right]^{\prime} \sin \theta_{j} e^{i m\left(\phi-\phi_{j}\right)} \\
& -\frac{i \mu_{\phi_{j}}}{r_{j} \sin \theta_{j}} \sum_{\ell=0}^{\infty} \sum_{m=-\ell}^{\ell} \frac{r_{<}^{\ell}}{r_{>}^{\ell+1}} \frac{(\ell-m) !}{(\ell+m) !} P_{\ell}^{m}(\cos \theta) \\
& \times P_{\ell}^{m}\left(\cos \theta_{j}\right) m e^{i m\left(\phi-\phi_{j}\right)}, \quad j=D, A
\end{aligned}
$$


where the dipole is located at $\left(r_{j}, \theta_{j}, \phi_{j}\right)$ with dipole moment components $\left(\mu_{r_{j}}, \mu_{\theta_{j}}, \mu_{\phi_{j}}\right),\left[P_{\ell}^{m}(x)\right]^{\prime} \equiv \frac{d P_{\ell}^{m}(x)}{d x}$ and $r_{<}\left(r_{>}\right)$ $=\min (\max )\left\{r, r_{j}\right\}$.

Hence, from matching the boundary conditions according to Fuchs and co-workers, ${ }^{19,20}$ we obtain $\widetilde{\Phi}_{\ell m j}$ in Eqs. (5), (12), and (19) given by

$$
\begin{aligned}
\tilde{\Phi}_{\ell m j}(r) & =\mu_{r_{j}} \sqrt{\frac{4 \pi}{2 \ell+1} \frac{(\ell-m) !}{(\ell+m) !}} \frac{\partial}{\partial r_{j}}\left(\frac{r_{<}^{\ell}}{r_{>}^{\ell+1}}\right) P_{\ell}^{m}\left(\cos \theta_{j}\right) e^{-i m \phi_{j}} \\
& -\frac{\mu_{\theta_{j}}}{r_{j}} \sqrt{\frac{4 \pi}{2 \ell+1} \frac{(\ell-m) !}{(\ell+m) !}} \frac{r_{<}^{\ell}}{r_{>}^{\ell+1}}\left[P_{\ell}^{m}\left(\cos \theta_{j}\right)\right]^{\prime} \sin \theta_{j} e^{-i m \phi_{j}} \\
& -\frac{i \mu_{\phi_{j}}}{r_{j} \sin \theta_{j}} \sqrt{\frac{4 \pi}{2 \ell+1} \frac{(\ell-m) !}{(\ell+m) !}} \frac{r_{<}^{\ell}}{r_{>}^{\ell+1}} P_{\ell}^{m}\left(\cos \theta_{j}\right) m e^{-i m \phi_{j}}, \quad j=D, A .
\end{aligned}
$$

Note that the above results have all assumed the dipole (D or A) to be located in vacuum. If any of these is in a dielectric medium (with constant $\varepsilon^{\prime}$ ), the above expression must be multiplied by a factor $\frac{1}{\varepsilon^{\prime}}$.

*Corresponding author. On leave from Department of Physics, Portland State University, P.O. Box 751, Portland, Oregon 972070751, USA.

${ }^{1}$ T. Förster, Ann. Phys. 437, 55 (1948); Discuss. Faraday Soc. 27, 7 (1959).

${ }^{2}$ D. L. Dexter, J. Chem. Phys. 21, 836 (1953).

${ }^{3}$ L. Stryer, Annu. Rev. Biochem. 47, 819 (1978).

${ }^{4}$ J. Zhang, Y. Fu, and J. R. Lakowicz, J. Phys. Chem. C 111, 50 (2007).

${ }^{5}$ P. Andrew and W. L. Barnes, Science 290, 785 (2000).

${ }^{6}$ P. Andrew and W. L. Barnes, Science 306, 1002 (2004).

${ }^{7}$ J. I. Gersten and A. Nitzan, Chem. Phys. Lett. 104, 31 (1984).

${ }^{8}$ X. M. Hua, J. I. Gersten, and A. Nitzan, J. Chem. Phys. 83, 3650 (1985).

${ }^{9}$ J. Malicka, Ignacy Gryczynski, Jiyu Fang, Jozef Kusba, and Joseph R. Lakowicz, Anal. Biochem. 315, 160 (2003); J. R. Lakowicz, Józef Kuśba, Yibing Shen, Joanna Malicka, Sabato D’Auria, Zygmunt Gryczynski, and Ignacy Gryczynski, J. Fluoresc. 13, 69 (2003).

${ }^{10}$ J. Zhang, Y. Fu, M. H. Chowdhury, and J. R. Lokawicz, J. Phys. Chem. C 111, 11784 (2007).

${ }^{11}$ J. Lindberg, K. Lindfors, T. Setälä, and M. Kaivola, J. Opt. Soc. Am. A 24, 3427 (2007).

${ }^{12}$ A. O. Govorov, J. Lee, and N. A. Kotov, Phys. Rev. B 76, 125308 (2007).

${ }^{13}$ H. Mertens, A. F. Koenderink, and A. Polman, Phys. Rev. B 76, 115123 (2007).

${ }^{14}$ Fully quantum mechanical approach using density-functional theory has been applied to treat ultrasmall metallic particles and shells in the literature; but these are often limited to sizes not greater than $\sim 1-2 \mathrm{~nm}$. See, e.g., W. Ekardt, Phys. Rev. B 32, 1961 (1985); 34, 526 (1986).

${ }^{15}$ K. Cho, Optical Response of Nanostructures (Springer-Verlag, Berlin, 2003).
${ }^{16}$ F. J. García de Abajo, J. Phys. Chem. C 112, 17983 (2008).

${ }^{17}$ See, e.g., S. J. Oldenburg, R. D. Averitt, S. L. Westcott, and N. J. Halas, Chem. Phys. Lett. 288, 243 (1998); S. L. Westcott, J. B. Jackson, C. Radloff, and N. J. Halas, Phys. Rev. B 66, 155431 (2002); E. Hao, S. Li, R. C. Bailey, S. Zou, G. C. Schartz, and J. T. Hupp, J. Phys. Chem. B 108, 1224 (2004); For a recent review, especially on biomedical applications of nanoshells, see L. R. Hirsch, Andre M. Gobin, Amanda R. Lowery, Felicia Tam, Rebekah A. Drezek, Naomi J. Halas, and Jennifer L. West, Ann. Biomed. Eng. 34, 15 (2006).

${ }^{18}$ M. Durach, A. Rusina, V. I. Klimov, and M. I. Stockman, New J. Phys. 10, 105011 (2008).

${ }^{19}$ R. Fuchs and F. Claro, Phys. Rev. B 35, 3722 (1987). Note that in the spirit of the additional boundary conditions, the four conditions here involve the continuity of the potentials and the displacement components in the form $\left.\Phi\right|_{r \rightarrow a_{-}}=\left.\Phi\right|_{r \rightarrow a_{+}}$and $\left.\Phi\right|_{r \rightarrow b_{-}}=\left.\Phi\right|_{r \rightarrow b_{+}} ;\left.\widetilde{\varepsilon} \frac{\partial \Phi}{\partial r}\right|_{r \rightarrow a_{-}}=\left.\frac{\partial \Phi_{D i s}}{\partial r}\right|_{r \rightarrow a_{+}}$and $\left.\widetilde{\varepsilon} \frac{\partial \Phi}{\partial r}\right|_{r \rightarrow b_{+}}=\left.\frac{\partial \Phi_{D i s}}{\partial r}\right|_{r \rightarrow b_{-}}$, where $\widetilde{\varepsilon}=\varepsilon_{0}$ or $\varepsilon^{\prime}$ depending on which of the three cases in Fig. 1 is considered.

${ }^{20}$ R. Rojas, F. Claro, and R. Fuchs, Phys. Rev. B 37, 6799 (1988).

${ }^{21}$ R. Chang and P. T. Leung, Phys. Rev. B 73, 125438 (2006); 75, 079901(E) (2007).

${ }^{22}$ N. K. Grady, N. J. Halas, and P. Nordlander, Chem. Phys. Lett. 399, 167 (2004).

${ }^{23}$ Z. E. Goude and P. T. Leung, Solid State Commun. 143, 416 (2007).

${ }^{24}$ A. T. Georges, Opt. Commun. 188, 321 (2001). Note that there exist other approaches in nonlocal optics which provide smooth charge density profile at surfaces; for spherical particles, see, e.g., P. Apell, Phys. Scr. 29, 146 (1984), and reference therein.

${ }^{25}$ E. Dulkeith, A. C. Morteani, T. Niedereichholz, T. A. Klar, J. Feldmann, S. A. Levi, F. C. J. M. van Veggel, D. N. Reinhoudt, M. Möller, and D. I. Gittins, Phys. Rev. Lett. 89, 203002 (2002); Nano Lett. 5, 585 (2005). 